\title{
Improved 8- and 16-State Space-Time Codes for 4PSK with Two Transmit Antennas
}

\author{
D. Mihai Ionescu, K. Kiran Mukkavilli, Zhiyuan Yan, and Jorma Lilleberg
}

\begin{abstract}
New space-time codes for 4PSK constellations, designed via a modified determinant criterion, send $2 \mathrm{~b} / \mathrm{s} / \mathrm{Hz}$ and show improved performance in quasistatic flat fading.

Keywords - Diversity methods, Fading channels, Trellis coded modulation.
\end{abstract}

\section{INTRODUCTION}

Space-time coding provides a means for achieving diversity in fading channels by implementing both space and time redundancy at the transmitter. While spacetime coding implies, in general, encoding both across time and across antennas, this letter addresses the problem of constructing good trellis space-time codes in the setting when all transmit antennas use the same $M$-PSK complex modulator constellation $\mathcal{M}$ of unit average energy, and exactly $\log _{2} M$ bits are transmitted during each multiple-channel use -i.e. coding redundancy is distributed in space. Extensions of the construction discussed herein to non- $M$-PSK constellations are natural. Consider a system with $L$ transmit antennas and $N$ receive antennas, designed so that fading across antennas be uncorrelated. Let $l$ be the number of symbol epochs - with respect to an arbitrary transmit antenna-required to send a codeword (a frame in [6]). If $l$ is regarded as the number of adjacent symbol epochs processed, to some extent, together in the detector, then a codeword is the concatenation of all symbols sent over all of the $L$ antennas during the corresponding $l$ consecutive symbol epochs; e.g., a generic codeword $c$ starting at discrete time instant $\kappa$ is $\boldsymbol{c}=\left[c_{\kappa}^{(1)} c_{\kappa}^{(2)} \ldots c_{\kappa}^{(L)} c_{\kappa+1}^{(1)} \ldots c_{\kappa+1}^{(L)} \ldots c_{\kappa+l-1}^{(1)} \ldots c_{\kappa+l-1}^{(L)}\right]^{T}$, where $c_{k}^{(i)}$ is a complex symbol from $\mathcal{M}$ transmitted at discrete time $k$ over transmit antenna $i$. A more meaningful representation for the codeword $c$ is the code matrix

$$
\boldsymbol{D}_{\boldsymbol{c}}=\left[\begin{array}{cccc}
c_{\kappa}^{(1)} & c_{\kappa}^{(2)} & \cdots & c_{\kappa}^{(L)} \\
\vdots & \vdots & \ddots & \vdots \\
c_{\kappa+l-1}^{(1)} & c_{\kappa+l-1}^{(2)} & \cdots & c_{\kappa+l-1}^{(L)}
\end{array}\right] .
$$

For two codewords $\boldsymbol{c}, \boldsymbol{e}$, let the code difference matrix be $\boldsymbol{D}_{\boldsymbol{e}}=\boldsymbol{D}_{\boldsymbol{e}}-\boldsymbol{D}_{\boldsymbol{c}}$. Let any transmitted symbol have energy $E_{s}$. Let the sequence of complex channel coefficients between transmit antenna $i$ and receive antenna $j$, denoted $\alpha_{i, j}(\cdot)$, be white complex Gaussian with $E\left\{\left|\alpha_{i, j}\right|^{2}\right\}=1, \forall i, j$. Assume that each $\alpha_{i, j}(\cdot)$ is constant over one frame (quasistatic flat fading) and that the $\alpha_{i, j}(\cdot)$ sequences are uncorrelated across antennas. Let $\gamma_{k}^{(i, j)}=\sqrt{E_{s}} \alpha_{i, j}[k]$. At receive antenna $j$ the sampled version of the signal received during a frame is $x_{k}^{(j)}=\sum_{i=1}^{L} \alpha_{i, j}[k] c_{k}^{(i)} \sqrt{E_{s}}+\eta_{k}^{(j)}$, $k=\kappa, \ldots, \kappa+l-1$, and becomes, in quasistatic flat fading
$(\mathrm{QFF})$,

$$
x_{k}^{(j)}=\sum_{i=1}^{L} c_{k}^{(i)} \gamma^{(i, j)}+\eta_{k}^{(j)},
$$

where $\eta_{k}^{(j)}$ is complex Gaussian noise with variance $N_{0} / 2$ per dimension. In $[6]$ it was shown that $\operatorname{Pr}(\boldsymbol{c} \mapsto \boldsymbol{e})$, the pairwise error probability (PEP) - of transmitting $c$ and deciding in favor of $\boldsymbol{e}$-is upper bounded via $\operatorname{Pr}(\boldsymbol{c} \mapsto \boldsymbol{e}) \leq$ $\left(\prod_{i=1}^{r} \lambda_{i}(\boldsymbol{c}, \boldsymbol{e})\right)^{-N}\left(E_{s} /\left(4 N_{0}\right)\right)^{-r N}$, where $r$ and $\lambda_{i}(\boldsymbol{c}, \boldsymbol{e})$ are, respectively, the rank and the nonzero eigenvalues of $\boldsymbol{D}_{\boldsymbol{e} c}^{\dagger} \boldsymbol{D}_{\boldsymbol{e c}}$. In quasistatic fading, the minimumover all codeword pairs - of the exponent $r N$ of $E_{s} / N_{0}$ is the diversity gain, and the minimum over all $\boldsymbol{c}, \boldsymbol{e}$ of $\left(\prod_{i=1}^{r} \lambda_{i}(\boldsymbol{c}, \boldsymbol{e})\right)^{1 / r}$ is the coding gain; the maximization of each is deemed desirable, respectively, by the rank and determinant criteria [6]. In fast fading the role of $r$ is assumed by the minimum symbol Hamming distance (MSHD), while a product distance determines the coding gain in place of the eigenvalue product [6]. In [4], [5] it was shown that the determinant criterion can be strengthened by requiring that the eigenvalues of $\boldsymbol{D}_{\boldsymbol{e c}}^{\dagger} \boldsymbol{D}_{\boldsymbol{e c}}$ be as close to each other as possible, for any codeword pair $\boldsymbol{c}, \boldsymbol{e}$. It can be shown [5] that this condition maximizes the product distance, given the Euclidean distance between $\boldsymbol{c}$ and $\boldsymbol{e}$. This modified determinat criterion is applied to construct codes for space-time trellis coded modulation (STTCM), which use 4PSK constellations and send $2 \mathrm{~b} / \mathrm{s} / \mathrm{Hz}$ by encoding over two modulator symbol epochs at a time, similar to the approach taken in multiple trellis coded modulation [3]. The design presented herein does not observe the various gain parameters from $[7]$ e.g. $\eta_{A P}(\cdot), \eta_{C P}(\cdot)$.

\section{Review of a Modified Determinant Criterion}

The equal eigenvalue criterion [4], [5] states that in i.i.d. $L$-transmit-antenna Rayleigh fading with perfect channel state information (CSI), an upper bound to the PEP is made as small as possible iff, for all pairs $\boldsymbol{D}_{\boldsymbol{c}}, \boldsymbol{D}_{\boldsymbol{e}}$, the Euclidean squared distances $\operatorname{tr}\left(\boldsymbol{D}_{\boldsymbol{e c}}^{\dagger} \boldsymbol{D}_{\boldsymbol{e c}}\right)$ are made as large as possible and the non-square matrices $\boldsymbol{D}_{\boldsymbol{e c}}$ behave as unitary matrices - up to appropriate proportionality factorsin the sense that $\boldsymbol{D}_{\boldsymbol{e c}}^{\dagger} \boldsymbol{D}_{\boldsymbol{e c}}=\left(\operatorname{tr}\left(\boldsymbol{D}_{\boldsymbol{e c}}^{\dagger} \boldsymbol{D}_{\boldsymbol{e c}}\right) / L\right) \boldsymbol{I}_{L}$. Suboptimal codes should be characterized by matrices $\boldsymbol{D}_{\boldsymbol{e c}}^{\dagger} \boldsymbol{D}_{\boldsymbol{e c}}$ whose main diagonal elements are as close as possible both to each other and to $\operatorname{tr}\left(\boldsymbol{D}_{\boldsymbol{e} c}^{\dagger} \boldsymbol{D}_{\boldsymbol{e c}}\right) / L$, and for which the rowwise sum of the absolute values of the elements off the main diagonal is as small as possible for each row. Essentially, for any pair $\boldsymbol{c}, \boldsymbol{e}$, one should enlarge the Euclidean distance between $\boldsymbol{c}, \boldsymbol{e}$ while rendering the eigenvalues of the square matrix $\boldsymbol{D}_{\boldsymbol{e c}}^{\dagger} \boldsymbol{D}_{\boldsymbol{e c}}$ to be equal, or as close as possible; the latter is equivalent to $\boldsymbol{D}_{\boldsymbol{e c}}^{\dagger} \boldsymbol{D}_{\boldsymbol{e c}}$ being as close to a diagonal matrix as possible. Necessarily, diversity is maximized in 
quasistatic fading (rank of $\boldsymbol{D}_{e c}^{\dagger} \boldsymbol{D}_{e c}$ is $L$ ). The proportionality of $\operatorname{tr}\left(\boldsymbol{D}_{\boldsymbol{e c}}^{\dagger} \boldsymbol{D}_{\boldsymbol{e c}}\right)$ to the Euclidean distance between $\boldsymbol{c}$ and $\boldsymbol{e}$ is crucial for identifying a constellation partitioning that is characteristic of trellis coded modulation schemes.

In order to enforce the desirable equal singular value (ESV) structure to valid $l \times L$ matrices $\boldsymbol{D}_{e c}$ it suffices to enforce it on submatrices. Suppose that $L$ divides $l$. Let $\boldsymbol{D}_{\boldsymbol{c}}$, $\boldsymbol{D}_{\boldsymbol{e}}, \boldsymbol{D}_{\boldsymbol{e}}$ be viewed as block vectors, i.e. $(l / L) \times 1$ matrices whose entries are $L \times L$ sub-matrices with elements from $\mathcal{M}$. Then any code matrix can be regarded as a sequence of $l / L, L \times L$ square sub-matrices, resulting from the unfolding of a trellis whose branches span, each, $L$ modulator symbol epochs, with each branch labeled by a valid $L \times L$ sub-matrix. A path through the trellis is selected as a function of the current state and a block of new input symbols. The set of all $L \times L$ matric building blocks can be regarded as a super-constellation. If these constituent blocks have the property that the Gram matrix of any valid pairwise difference is optimal - or close to optimal - then the properties mentioned above are transfered from $\boldsymbol{D}_{\boldsymbol{c}}, \boldsymbol{D}_{\boldsymbol{e}}$ to $\boldsymbol{D}_{\boldsymbol{e}}$. For $L=2$ and 4 PSK, the 16 orthogonal complex matrices discussed by Alamouti [1] do have the aforementioned ESV structure (optimal) for pairwise differences. However, in order to achieve the desired $\log _{2} M=2 \mathrm{~b} / \mathrm{s} / \mathrm{Hz}$ one must have enough $2 \times 2$ constituent matrices in the super-constellation; this requires augmenting the optimal matric set - e.g., by a reflection of itself, see Table II - to the effect that some codematrix pairs in the augmented set will not obey the ESV structure. The design goal becomes insuring that difference code matrices pertaining to an error event path (EEP) of length $k \leq k^{\prime}$ transitions ( $k L$ modulator symbols) be optimal for $k^{\prime}$ as large as possible, and as close to optimal as feasible for $k>k^{\prime}$. Note that Alamouti's transmit diversity scheme [1] for $L=2$ transmit antennas can be employed by simply appending, to some encoder's output, a mapper from encoded symbols to constellation points, followed by a Hurwitz-Radon transformer applied to two consecutive complex symbols. This provides only diversity gain and is not the approach taken herein. It is worth realizing that Alamouti's scheme with 4PSK and two transmit antennas over additive white Gaussian noise (AWGN) has the same bit error probability as uncoded 4PSK in AWGN. In subsequent plots, Alamouti's scheme serves as a diversity two reference with no coding gain.

\section{New Space-Time Trellis Codes}

Consider the $L=2$ case and assume that each transmit antenna uses 4PSK modulation; other $M$-PSK constellations can be accommodated using similar steps. A trellis coded modulation scheme with $q$ states, where each trellis transition covers two symbols, can be obtained naturally by constructing a super-constellation whose points are $2 \times 2$ matrices chosen so as to facilitate the existence of the structure discussed above; the matrix elements are from a 4PSK constellation and there must be enough super-constellation points to allow the transmission of 2 bits per channel use. Table II shows 32 matrices $\boldsymbol{C}_{i}, i=0, \ldots, 31$, whose entries represent indices of complex points from the $4 \mathrm{PSK}$ constellation (Table I). Each $\boldsymbol{C}_{i}$ defines the 4PSK sym-

TABLE I

INDEXING FOR THE 4PSK CONSTELLATION POINTS.

\begin{tabular}{|c|c|c|c|}
\hline$s_{0}$ & $s_{1}$ & $s_{2}$ & $s_{3}$ \\
\hline \hline$\frac{1}{\sqrt{2}}+j \frac{1}{\sqrt{2}}$ & $-\frac{1}{\sqrt{2}}+j \frac{1}{\sqrt{2}}$ & $-\frac{1}{\sqrt{2}}-j \frac{1}{\sqrt{2}}$ & $\frac{1}{\sqrt{2}}-j \frac{1}{\sqrt{2}}$ \\
\hline
\end{tabular}

TABLE II

The $2 \times 2$ matrices $\boldsymbol{C}_{i}, i=0, \ldots, 31$, Along With RELEVANT Cosets $\mathcal{C}_{l}$ AND CORRESPONDING UNCODED BITS, VS. NUMBER OF STATES $q$.

\begin{tabular}{|c|c|c|c|c|c|c|c|c|c|c|c|}
\hline 0 & & $q$ & $=8$ & & $q$ & $=16$ & & $q$ & $=24$ & & \\
\hline .7 & 8 & 16 & 15 & 81 & \begin{tabular}{|c|}
16 \\
\end{tabular} & .23 & 8 & & . 31 & $\bar{\varepsilon}$ & \\
\hline & $\begin{array}{c}C_{0} \\
0\end{array}$ & $\begin{array}{c}\mathcal{C}_{0} \\
0\end{array}$ & $\left.\begin{array}{lll}3 & 3 \\
0 & 2\end{array}\right]$ & $\begin{array}{c}\mathcal{C}_{0} \\
01\end{array}$ & $\begin{array}{c}\mathcal{C}_{2} \\
1\end{array}$ & $\begin{array}{lll}3 & 1 \\
0 & 0\end{array}$ & $\begin{array}{l}\mathcal{C}_{5} \\
00\end{array}$ & $\begin{array}{c}\mathcal{C}_{8} \\
0\end{array}$ & $\begin{array}{l}\begin{array}{ll}1 & 1 \\
0 & 2\end{array}\end{array}$ & 01 & $\mathcal{C}_{10}$ \\
\hline 2 & $\begin{array}{l}\mathcal{C}_{1} \\
00\end{array}$ & $\begin{array}{c}\mathcal{C}_{1} \\
0\end{array}$ & $\begin{array}{l}32 \\
12\end{array}$ & $\begin{array}{l}\mathcal{C}_{1} \\
01\end{array}$ & $\begin{array}{c}\mathcal{C}_{3} \\
1\end{array}$ & $\begin{array}{ll}3 & 0 \\
1 & 0\end{array}$ & $\begin{array}{l}\mathcal{C}_{4} \\
00\end{array}$ & $\begin{array}{c}\mathcal{C}_{9} \\
0\end{array}$ & & $\begin{array}{l}\mathcal{C}_{4} \\
01\end{array}$ & $\begin{array}{c}\mathcal{C}_{11} \\
1\end{array}$ \\
\hline & $\begin{array}{c}\mathcal{C}_{0} \\
10\end{array}$ & $\begin{array}{c}\mathcal{C}_{2} \\
0\end{array}$ & & $\begin{array}{l}\mathcal{C}_{0} \\
11\end{array}$ & $\begin{array}{c}\mathcal{C}_{0} \\
1\end{array}$ & $\begin{array}{ll}3 & 3 \\
2 & 0\end{array}$ & $\begin{array}{l}\mathcal{C}_{5} \\
10\end{array}$ & $\left(\begin{array}{c}\mathcal{C}_{10} \\
0\end{array}\right.$ & $\begin{array}{ll}1 & 3 \\
2 & 2\end{array}$ & $\begin{array}{l}C_{5} \\
11\end{array}$ & $\begin{array}{c}\mathcal{C}_{8} \\
1\end{array}$ \\
\hline 0 & $\begin{array}{l}\mathcal{C}_{1} \\
10\end{array}$ & $\begin{array}{c}\mathcal{C}_{3} \\
0\end{array}$ & $\begin{array}{l}30 \\
32\end{array}$ & $\begin{array}{l}\mathcal{C}_{1} \\
11\end{array}$ & $\begin{array}{c}\mathcal{C}_{1} \\
1\end{array}$ & $\begin{array}{l}32 \\
30\end{array}$ & $\begin{array}{l}\mathcal{C}_{4} \\
10\end{array}$ & $\mid \begin{array}{c}\mathcal{C}_{11} \\
0\end{array}$ & $\begin{array}{l}12 \\
32\end{array}$ & 11 & $\begin{array}{c}\mathcal{C}_{9} \\
1\end{array}$ \\
\hline $\begin{array}{ll}0 & 3 \\
0 & 1\end{array}$ & $\mid \begin{array}{l}\mathcal{C}_{3} \\
00\end{array}$ & $\begin{array}{c}\mathcal{C}_{5} \\
0\end{array}$ & & $\begin{array}{l}\mathcal{C}_{3} \\
01\end{array}$ & $\begin{array}{c}\mathcal{C}_{7} \\
1\end{array}$ & $\begin{array}{lll}2 & 1 \\
0 & 1\end{array}$ & $\left|\begin{array}{l}\mathcal{C}_{6} \\
00\end{array}\right|$ & $\begin{array}{c}\mathcal{C}_{13} \\
0\end{array}$ & $\begin{array}{lll}0 & 1 \\
0 & 3\end{array}$ & & $\begin{array}{c}\mathcal{C}_{15} \\
1\end{array}$ \\
\hline $\begin{array}{ll}02 \\
11\end{array}$ & $\mid \begin{array}{l}\mathcal{C}_{2} \\
00\end{array}$ & $\begin{array}{c}\mathcal{C}_{4} \\
0\end{array}$ & {$\left[\begin{array}{ll}2 & 2 \\
1 & 3\end{array}\right.$} & $\begin{array}{l}\mathcal{C}_{2} \\
01\end{array}$ & $\begin{array}{c}\mathcal{C}_{6} \\
1\end{array}$ & $\begin{array}{l}20 \\
11\end{array}$ & $\left|\begin{array}{l}\mathcal{C}_{7} \\
00\end{array}\right|$ & $\mid \begin{array}{c}\mathcal{C}_{12} \\
0\end{array}$ & $\begin{array}{ll}0 & 0 \\
1 & 3\end{array}$ & & $\begin{array}{l}14 \\
1\end{array}$ \\
\hline 01 & $\begin{array}{l}\mathcal{C}_{3} \\
10\end{array}$ & $\begin{array}{c}\mathcal{C}_{7} \\
0\end{array}$ & 21 & $\begin{array}{l}\mathcal{C}_{3} \\
11\end{array}$ & $\begin{array}{c}\mathcal{C}_{5} \\
1\end{array}$ & $\begin{array}{l}23 \\
21\end{array}$ & $\mid \begin{array}{l}\mathcal{C}_{6} \\
10\end{array}$ & $\begin{array}{c}\mathcal{C}_{15} \\
0\end{array}$ & $\begin{array}{l}03 \\
23\end{array}$ & 11 & $\mathcal{C}_{13}$ \\
\hline & $\mathcal{C}_{2}$ & $\mathcal{C}_{6}$ & 20 & $\begin{array}{l}\mathcal{C}_{2} \\
11\end{array}$ & $\begin{array}{c}\mathcal{C}_{4} \\
1\end{array}$ & $\begin{array}{lll}2 & 2 \\
3 & 1\end{array}$ & $\left|\begin{array}{l}\mathcal{C}_{7} \\
10\end{array}\right|$ & $\begin{array}{c}\mathcal{C}_{14} \\
0\end{array}$ & $\left.\begin{array}{ll}0 & 2 \\
3 & 3\end{array}\right]$ & & $\mathcal{C}_{12}$ \\
\hline
\end{tabular}

TABLE III

Single Shift REgister IMPLEMENTATION OF THE COSET SELECTOR IS DESCRIBED By MATRIX $\boldsymbol{G}$, GIVEN VS. THE NUMBER OF STATES $q$.

\begin{tabular}{|c|c|c|}
\hline$q$ & 8 & 16 \\
\hline $\boldsymbol{G}^{T}$ & {$\left[\begin{array}{lllll}0 & 1 & 0 & 0 & 1 \\
1 & 0 & 0 & 1 & 0 \\
0 & 0 & 1 & 0 & 0\end{array}\right]$} & 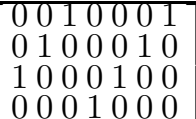 \\
\hline
\end{tabular}

bols to be sent over the $L=2$ transmit antennas, during two consecutive symbol epochs. The squared Euclidean distance between $\boldsymbol{C}_{i}$ and $\boldsymbol{C}_{j}$ is $\operatorname{tr}\left(\left(\boldsymbol{C}_{i}-\boldsymbol{C}_{j}\right)^{\dagger}\left(\boldsymbol{C}_{i}-\boldsymbol{C}_{j}\right)\right)$. The super-constellation is partitioned in the usual way producing, as a function of $q$, the appropriate cosets as shown also in Table II. The elements within one coset are distinguished by means of uncoded bits, via the mapping listed in Table II. Finally the convolutional code selecting the cosets is described in terms of a matrix $\boldsymbol{G}$, similar in structure with the matrices used to describe the convolutional STCs reported in [7], [2]. The new STTCM codes verify:

- The minimum Euclidean distance between any two branches leaving (arriving into) a given state is maximized. 
- $\boldsymbol{D}_{e c}^{\dagger} \boldsymbol{D}_{\boldsymbol{e c}}$ has equal eigenvalues for any $\boldsymbol{D}_{\boldsymbol{e c}}$ corresponding to EEPs of length $k \leq 2$ (i.e. up to four 4PSK symbols).

- The MSHD between any two parallel transitions is 2, thus the diversity order remains 2 in rapid fading [6], despite of parallel transitions. When the new 8- and 16-state STTCM codes are compared with, e.g., Tarokh's 16- and 32-state codes - equal complexity, respectively, - the latter have a MSHD of 3 hence exhibit higher diversity in rapid fading.

For 130 symbol frames, at equal spectral efficiency in QFF with perfect CSI, Figures 1, 2 compare the average frame error probability (FEP) of the new STTCM codes, several other trellis STCs [6], [2], [7] of correspondingly equal complexity, and Alamouti's scheme. Complexity is judged in terms of the product between the number of states and the number of transitions emerging from each state, normalized to one modulator symbol epoch; e.g., since each transition in the new, 8-state STTCM code covers two symbol epochs, and sixteen transitions (including parallel ones) emerge from each state, the complexity is $16 \times 8 / 2=64$ - equal to that of Tarokh's 16 -state trellis STC. Figure 3 compares the new 8-state STTCM code

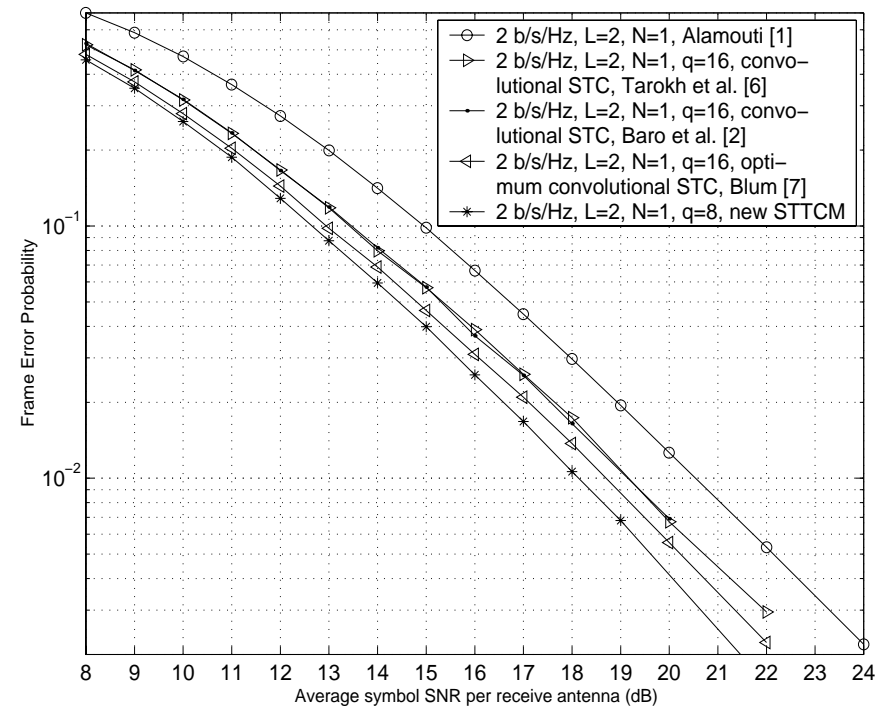

Fig. 1. Some $2 \mathrm{~b} / \mathrm{s} / \mathrm{Hz}, 4 \mathrm{PSK}$ STCs in quasistatic fading. New code and all 16-state convolutional STCs are equally complex.

with the same trellis STCs from [6], [2], [7], for $N=2$ receive antennas. When receive diversity is present, the new STTCM code performs worse than the optimum convolutional STC from [7] (by less than $0.3 \mathrm{~dB}$ ) and better than the other STCs. The performance-wise reversal between the first two codes - relative to the $N=1$ case - may be due to the distance spectrum of the new STTCM code, which was not optimized beyond insuring that all lengthtwo EEPs obey the ESV criterion.

\section{Conclusions}

New $2 \mathrm{~b} / \mathrm{s} / \mathrm{Hz}$ STCs for two transmit antennas and 4PSK constellations show improved performance in QFF with no receive diversity. Characterization, and optimization thereof, in terms of various gain parameters from [7] - e.g. $\eta_{A P}(\cdot), \eta_{C P}(\cdot)$ - are topics for future investigations.

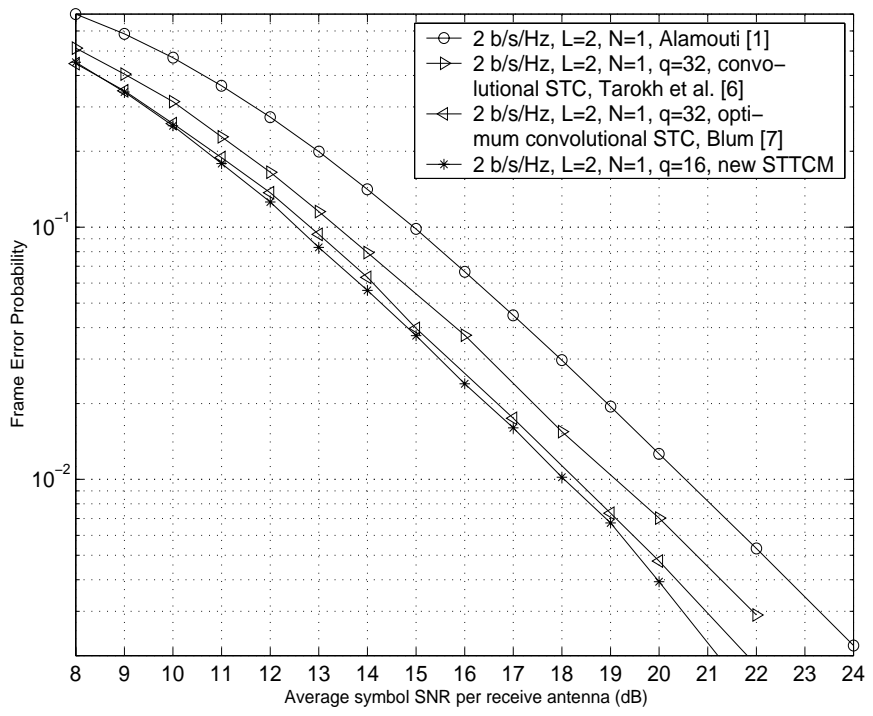

Fig. 2. Some $2 \mathrm{~b} / \mathrm{s} / \mathrm{Hz}, 4 \mathrm{PSK}$ STCs in quasistatic fading. New code and all 32-state convolutional STCs are equally complex.

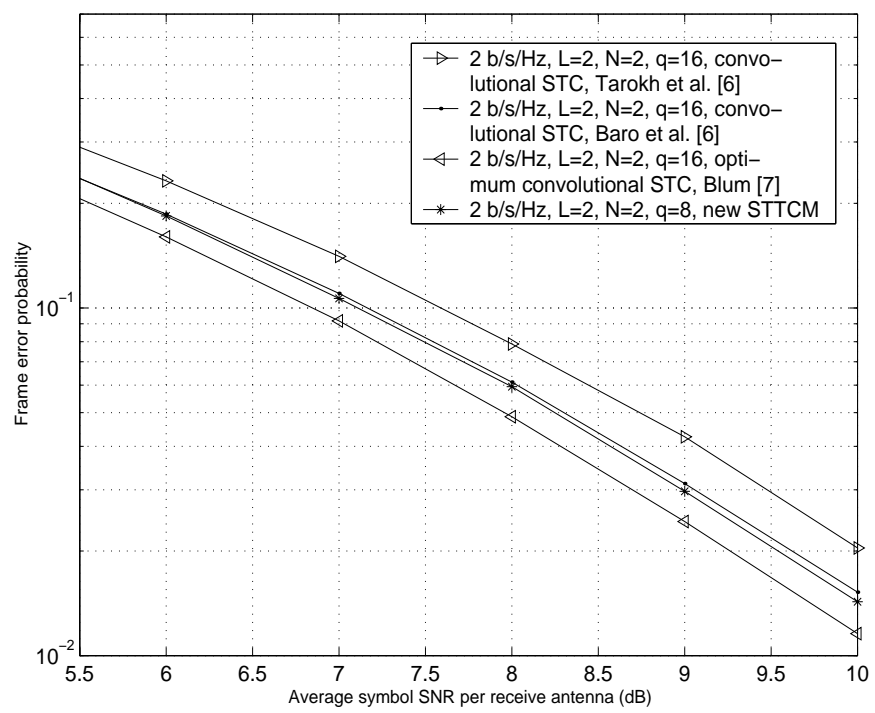

Fig. 3. Some $2 \mathrm{~b} / \mathrm{s} / \mathrm{Hz}$, 4PSK STCs in quasistatic fading. New code and all 16-state convolutional STCs are equally complex.

\section{REFERENCES}

[1] S. M. Alamouti, "A simple transmit diversity technique for wireless communications," IEEE J. Select. Areas Commun., vol. 16, pp. 1451-1458, Oct. 1998.

[2] S. Bäro, G. Bauch, and A. Hansmann, "Improved codes for space-time trellis-coded modulation," IEEE Commun. Lett., vol. 4, pp. 20-22, Jan. 2000.

[3] D. Divsalar, and M. K. Simon, "Multiple trellis coded modulation (MTCM)," IEEE Trans. Commun., pp. 410-418, Apr. 1988.

[4] D. M. Ionescu, "New results on space-time code design criteria," Proc. Wireless Commun. and Networking Conf., Sept. 1999.

[5] D. M. Ionescu, "On Space-Time Code Design for Optimal Performance in Both Gaussian and Fading Channels," submitted to IEEE J. Select. Areas in Commun.

[6] V. Tarokh, N. Seshadri, and A.R. Calderbank, "Space-time codes for high data rate wireless communication: Performance criteria and code construction," IEEE Trans. IT, March 1998.

[7] R. S. Blum, "Some Analytical Tools for the Design of SpaceTime Convolutional Codes," submitted to IEEE Trans. Inform. Theory; similar work in Proc. Conf. on Inf. Sciences and Syst., Princeton Univ., Princeton, NJ, March, 1999 